\title{
Multi-channel analog of the effective-range expansion
}

\author{
S. A. Rakityansky ${ }^{1)}$ and N. Elander ${ }^{2)}$ \\ 1) Dept. of Physics, University of Pretoria, Pretoria 0002, South Africa \\ 2) Div.of Molecular Physics, Dept. of Physics, Stockholm University, \\ Stockholm, SE-106 91, Sweden
}

August 15, 2018

\begin{abstract}
Similarly to the standard effective range expansion that is done near the threshold energy, we obtain a generalized power-series expansion of the multi-channel Jostmatrix that can be done near an arbitrary point on the Riemann surface of the energy within the domain of its analyticity. In order to do this, we analytically factorize its momentum dependencies at all the branching points on the Riemann surface. The remaining single-valued matrix functions of the energy are then expanded in the power-series near an arbitrary point in the domain of the complex energy plane where it is analytic. A systematic and accurate procedure has been developed for calculating the expansion coefficients. This means that near an arbitrary point in the domain of physically interesting complex energies it is possible to obtain a semi-analytic expression for the Jost-matrix (and therefore for the Smatrix) and use it, for example, to locate the spectral points (bound and resonant states) as the S-matrix poles.
\end{abstract}

PACS number(s): 03.65.Nk, 03.65.Ge, 24.30.Gd

Published in: J.Phys.A44(2011)115303

\section{Introduction}

Taylor-type power-series expansions are very common in physics. In quantum scattering theory, the most frequently used expansion of this kind is known as the effective-range 
expansion. In the case of a short-range potential, it represents the cotangent function of the scattering phase shift $\delta_{\ell}(k)$ in the form

$$
k^{2 \ell+1} \cot \delta_{\ell}(k)=\sum_{n=0}^{\infty} c_{\ell n} k^{2 n} .
$$

Here the right-hand side is a sum of terms proportional to even powers of the collision momentum $k$, and $c_{\ell n}$ are energy independent expansion coefficients. Originally (see, for example, Ref. [1] and Ref. [2] for a historical review), this expansion was suggested in nuclear physics for the $S$-wave nucleon-nucleon scattering in the form

$$
k \cot \delta_{0}(k)=-\frac{1}{a}+\frac{1}{2} r_{0} k^{2}-P r_{0}^{3} k^{4}+Q r_{0}^{5} k^{6}+\cdots,
$$

where the first two parameters on the right-hand side, namely, $a$ and $r_{0}$, were called the scattering length and the effective radius. The parameters in the higher terms of this expansion $(P, Q$, etc.) were known as the shape parameters. This name originated from their dependence on the shape of a chosen $N N$-potential, while $a$ and $r_{0}$ were mainly determined by the strength of the interaction and the range of the force.

Originally, some rather inconvenient integral formulae (see, for example, Ref. [2]) for calculating the parameters on the right hand side of Eq. (2) were suggested. The expansion, however, was mainly used to parametrize the experimental data by choosing appropriate values for the first few coefficients. It is still frequently used for such purposes not only in nuclear physics but also for parametrizing the low-energy collisions between atoms and molecules (see, for example, Refs. [3, 4, 5, 6, 7, 8]). The expansion is also useful for simplified analytical description of the scattering near the threshold energy. For example, in one of the recent works of this type the effective-range expansion is used to study the causality constraints on low-energy universality [9].

A workable and rather accurate method for calculating the low-energy parameters $a$, $r_{0}, P$, etc., was developed about fifty years ago [10, 11, 12, 13, 14] within the variablephase approach. Recently the same equations were re-derived in Ref. [15] using the technique of canonical quantization. In this method, the low-energy parameters are the asymptotic values $(r \rightarrow \infty)$ of the corresponding radial functions $a(r), r_{0}(r), P(r)$, etc., obeying a system of first-order differential equations. At a fixed $r$, these functions give the corresponding low-energy parameters for the potential which is cut off at this value of the radius. 
The main drawback of using the differential equations for $a(r), r_{0}(r), P(r)$, etc., is that such a method is inconvenient in the case of potentials supporting bound states. Indeed, with the radius increasing from zero to infinity, the potential grows from nothing to its complete shape and its bound states appear one by one at certain values of $r$. Each new bound state initially appears with zero binding energy which corresponds to infinite scattering length. Therefore the function $a(r)$ has singularities, which cause serious problems when the system of differential equations is solved numerically. This difficulty is avoided if instead of $a(r), r_{0}(r), P(r)$, etc., we consider the expansion coefficients of the Jost function for the potential cut off at $r$. These coefficients also obey a system of differential equations but do not have any singularities. The standard low-energy parameters are obtained from them via simple algebra [16].

The traditional approach has one significant limitation, namely, the effective-range expansion is only applicable near the point $k=0$, i.e. when the energy is close to the threshold. In Ref. [16], the expansion (11) is generalized by writing it as a series of powers of $\left(k-k_{0}\right)$ with an arbitrary complex $k_{0}$. By doing this, it is possible, for example, to explore the complex $k$-plane in search for resonances. When $k_{0} \neq 0$, the generalized effective-range expansion is no longer a low-energy approximation. In other words, instead of making the expansion around the threshold, one can do it around any point in the complex plane.

Yet another limitation of the original expansion (1) is that it is formulated for a single-channel problem. For multi-channel problems, something similar was also suggested $[17,18,19,20,21,22$. In these papers, the authors tried to keep as much similarity with the single-channel case as possible. Their adherence to the functions of the type $\cot \delta$ limited the flexibility and clarity of the suggested equations.

In the present paper, we use a different approach in generalizing the effective-range expansion to multi-channel problems. Instead of considering the channel phase shifts and their cotangents, we look at the problem from a more general point of view. As a first step, we analyze the structure of the Jost matrix and explicitly factorize the dependencies of its matrix elements on odd powers of the channel momenta. These factors determine all the branching points of the Riemann surface of the energy, while the remaining factors are single-valued holomorphic functions defined on a simple energy plane. These functions are then expanded in power series of $\left(E-E_{0}\right)$, where $E_{0}$ is an arbitrary complex energy. As a result, we obtain an expression for the Jost matrix such that each of its elements is a product of a nonanalytic "branching" factor and a power series. In this way, we are not limited to the threshold points, but expand the Jost matrix practically anywhere on 
the Riemann surface. When the Jost matrix is obtained in such a semi-analytic form (the first several terms of the expansion), the $S$ matrix and all the observables can be easily calculated. Although it is possible, there is no need in introducing the generalized scattering length and other parameters. The Jost matrix expansion coefficients are more simple, clear, and convenient for this purpose.

\section{Multi-channel Schrödinger problem}

Consider a quantum mechanical two-body problem which, after separation of the motion of its center of mass, is reduced to an effective problem of one body whose dynamics is governed by the Hamiltonian

$$
H=H_{0}+\mathcal{U}+h
$$

consisting of the free-motion part $H_{0}$, the interaction operator $\mathcal{U}$, and the Hamiltonian $h$ that describes the internal dynamics in the moving body (for example, the internal states of colliding atoms). Each internal state of the body corresponds to a different channel of the scattering process. In general, there are infinite number of the internal states, i.e. the eigenstates of $h$,

$$
h|n\rangle=E_{n}|n\rangle, \quad n=1,2,3, \ldots \infty
$$

We assume that only $N$ internal states are important. In other words, we approximate the internal Hamiltonian by the $N$ terms

$$
h \approx \sum_{n=1}^{N}|n\rangle E_{n}\langle n|
$$

The total Hamiltonian taken in the representation of the relative coordinate $\vec{r}$ and sandwiched between $\langle n|$ and $\left|n^{\prime}\right\rangle$, becomes the $N \times N$ matrix

$$
H_{n n^{\prime}}=-\delta_{n n^{\prime}} \frac{\hbar^{2}}{2 \mu_{n}} \Delta_{\vec{r}}+\mathcal{U}_{n n^{\prime}}(\vec{r})+E_{n} \delta_{n n^{\prime}}
$$

where $\mu_{n}$ is the reduced mass in the channel $n$. Therefore the eigenvalue equation $H \Psi=$ $E \Psi$ is reduced to a set of coupled differential equations for the channel wave functions

$$
\left[\frac{\hbar^{2}}{2 \mu_{n}} \Delta_{\vec{r}}+\left(E-E_{n}\right)\right] \psi_{n}(E, \vec{r})=\sum_{n^{\prime}=1}^{N} \mathcal{U}_{n n^{\prime}}(\vec{r}) \psi_{n^{\prime}}(E, \vec{r})
$$


The eigenenergies $E_{n}$ of the internal Hamiltonian $h$ are the thresholds for the corresponding channels. An eigenstate of the Hamiltonian (6), corresponding to the eigenvalue $E$, is a column-matrix

$$
\Psi(E, \vec{r})=\left(\begin{array}{c}
\psi_{1}(E, \vec{r}) \\
\psi_{2}(E, \vec{r}) \\
\vdots \\
\psi_{N}(E, \vec{r})
\end{array}\right)
$$

where each row describes the relative motion in the corresponding channel.

To avoid unnecessary complicated notation, we assume that the relative motion in each channel has a single value $\ell_{n}$ of the angular momentum. This does not compromise the generality of our consideration. Indeed, we can always treat the states with different values of angular momentum (or of any other quantum number) as different channels, but perhaps with the same threshold energies.

Therefore the angular dependence of the channel wave function can be factorized in the simple way

$$
\psi_{n}(E, \vec{r})=\frac{u_{n}(E, r)}{r} Y_{\ell_{n} m_{n}}(\theta, \varphi) .
$$

This reduces Eq. (7) to the system of coupled equations for the radial parts of the channel wave functions,

$$
\left[\partial_{r}^{2}+k_{n}^{2}-\frac{\ell_{n}\left(\ell_{n}+1\right)}{r^{2}}\right] u_{n}(E, \vec{r})=\sum_{n^{\prime}=1}^{N} V_{n n^{\prime}}(r) u_{n^{\prime}}(E, \vec{r})
$$

where

$$
V_{n n^{\prime}}(r)=\frac{2 \mu_{n}}{\hbar^{2}} \int Y_{\ell_{n} m_{n}}^{*}(\theta, \varphi) \mathcal{U}_{n n^{\prime}}(\vec{r}) Y_{\ell_{n^{\prime}} m_{n^{\prime}}}(\theta, \varphi) d \Omega_{\vec{r}}
$$

and the channel momenta are defined as

$$
k_{n}=\sqrt{\frac{2 \mu_{n}}{\hbar^{2}}\left(E-E_{n}\right)} .
$$

In what follows, we assume that the interaction potential is non-singular and of short range, i.e. that all its matrix elements are less singular than $1 / r^{2}$ at the origin and exponentially vanish at infinity. In principle, the class of acceptable potentials can be much wider. This however would require a complicated analysis of the analytic properties of the 
multi-channel Jost matrix in order to identify the domain of complex energies where the Jost matrix can be analytically continued to. With the exponentially decaying potentials, we can rely on such an analysis given in Ref. [23] where the domain of analyticity of the multi-channel $T$-matrix and $S$-matrix is rigorously established. Therefore by narrowing the class of the potentials, we sacrifice the generality for the sake of clarity.

The boundary conditions for Eqs. (10) are derived from the requirement that any physical solution must be regular at the point $r=0$ and have special behaviour when $r \rightarrow \infty$, which is different for bound, resonant, and scattering states. In Sect. 4, we consider these conditions in more detail.

\section{Multi-channel Jost matrix}

A system of $N$ linear second-order differential equations of the type (10) has $2 N$ linearly independent column-solutions and only half of them are regular at the origin (see, for example, Ref. [24]). Combining these regular columns in a square matrix, we obtain the so called fundamental matrix of the regular solutions,

$$
\Phi(E, r)=\left(\begin{array}{cccc}
\phi_{11}(E, r) & \phi_{12}(E, r) & \cdots & \phi_{1 N}(E, r) \\
\phi_{21}(E, r) & \phi_{22}(E, r) & \cdots & \phi_{2 N}(E, r) \\
\vdots & \vdots & \vdots & \vdots \\
\phi_{N 1}(E, r) & \phi_{N 2}(E, r) & \cdots & \phi_{N N}(E, r)
\end{array}\right) .
$$

Any other regular solution can only be a linear combination of the columns of this fundamental matrix. In particular, any physical solution of Eqs. (10) is a linear combination of the columns of the corresponding fundamental matrix,

$$
\left(\begin{array}{c}
u_{1} \\
u_{2} \\
\vdots \\
u_{N}
\end{array}\right)=C_{1}\left(\begin{array}{c}
\phi_{11} \\
\phi_{21} \\
\vdots \\
\phi_{N 1}
\end{array}\right)+C_{2}\left(\begin{array}{c}
\phi_{12} \\
\phi_{22} \\
\vdots \\
\phi_{N 2}
\end{array}\right)+\cdots+C_{N}\left(\begin{array}{c}
\phi_{1 N} \\
\phi_{2 N} \\
\vdots \\
\phi_{N N}
\end{array}\right)
$$

This guaranties its correct behaviour when $r \rightarrow 0$. As far as the asymptotic behaviour $(r \rightarrow \infty)$ is concerned, its correct form can be achieved by proper choice of the combination coefficients $C_{n}$. 
Since the potential is of a short range, far away from the origin the right hand sides of Eqs.(10) vanish and these equations decouple,

$$
\left[\partial_{r}^{2}+k_{n}^{2}-\frac{\ell_{n}\left(\ell_{n}+1\right)}{r^{2}}\right] u_{n}(E, \vec{r}) \approx 0, \text { when } \quad r \rightarrow \infty .
$$

These are the Riccati-Bessel equations. As a pair of linearly independent solutions of each of them, we can take the Riccati-Hankel functions $h_{\ell_{n}}^{( \pm)}\left(k_{n} r\right)$.

Despite the fact that equations (15) are decoupled, we can still treat them as a system. It therefore has $2 N$ linearly independent column-solutions which can be chosen in many different ways. The most convenient choice is the following set of $2 N$ columns grouped in two square matrices

$$
\begin{aligned}
W^{(\mathrm{in})} & =\left(\begin{array}{cccc}
h_{\ell_{1}}^{(-)}\left(k_{1} r\right) & 0 & \cdots & 0 \\
0 & h_{\ell_{2}}^{(-)}\left(k_{2} r\right) & \cdots & 0 \\
\vdots & \vdots & \vdots & \vdots \\
0 & 0 & \vdots & h_{\ell_{N}}^{(-)}\left(k_{N} r\right)
\end{array}\right) \\
W^{(\text {out })} & =\left(\begin{array}{cccc}
h_{\ell_{1}}^{(+)}\left(k_{1} r\right) & 0 & \cdots & 0 \\
0 & h_{\ell_{2}}^{(+)}\left(k_{2} r\right) & \cdots & 0 \\
\vdots & \vdots & \vdots & \vdots \\
0 & 0 & \vdots & h_{\ell_{N}}^{(+)}\left(k_{N} r\right)
\end{array}\right)
\end{aligned}
$$

that represent the in-coming and out-going spherical waves in all the channels. These $2 N$ columns form a basis in the space of solutions. In other words, any column-solution of Eq. (15) is a linear combination of these $2 N$ columns. In particular, each column of matrix (13) at large distances becomes such a combination. The combination coefficients have two subscripts: one to indicate which column of (13) is expanded and the other is the summation subscript. Similarly to Eqs. (16, 17), we can group these coefficients in square matrices (they depend on the choice of the energy, but do not depend on $r$ ). Thus we have

$$
\Phi(E, r) \underset{r \rightarrow \infty}{\longrightarrow} W^{(\text {in })}(E, r) F^{(\text {in })}(E)+W^{(\text {out })}(E, r) F^{(\text {out })}(E),
$$

where, by analogy with the single-channel case (see, for example, Ref. [25]), the energydependent matrices $F^{\text {(in/out) }}(E)$ can be called Jost matrices. It is not difficult to show that they determine the $S$-matrix

$$
S(E)=F^{(\text {out })}(E)\left[F^{(\text {in })}(E)\right]^{-1}
$$


and thus give complete description of the underlying physical system. The spectral points $E=\mathcal{E}_{n}$ (bound states and resonances) are those where the inverse matrix $\left[F^{(\mathrm{in})}(E)\right]^{-1}$ does not exist, i.e. the points where

$$
\operatorname{det} F^{(\text {in) }}\left(\mathcal{E}_{n}\right)=0
$$

\section{Transformation of the Schrödinger equation}

At large distances, the fundamental regular matrix $\Phi(E, r)$ is a linear combination (18) with the $r$-independent coefficient matrices $F^{(\text {in/out })}(E)$. We can, however, look for $\Phi(E, r)$ in the same form at any point $r$, but with the coefficient matrices depending on $r$,

$$
\Phi(E, r) \equiv W^{(\text {in })}(E, r) \mathcal{F}^{(\text {in })}(E, r)+W^{\text {(out) }}(E, r) \mathcal{F}^{(\text {out })}(E, r) .
$$

Now, instead of one unknown function $\Phi(E, r)$, we have two unknown functions, $\mathcal{F}^{(\mathrm{in})}(E, r)$ and $\mathcal{F}^{\text {(out) }}(E, r)$, which therefore cannot be independent of each other. In principle, we can arbitrarily impose any (reasonable) additional condition relating them. The most convenient is to demand that

$$
W^{(\text {in })}(E, r) \frac{\partial}{\partial r} \mathcal{F}^{(\text {in })}(E, r)+W^{(\text {out })}(E, r) \frac{\partial}{\partial r} \mathcal{F}^{(\text {out })}(E, r)=0
$$

which is standard in the theory of differential equations, and is known as the Lagrange condition within the variation parameters method [26]. Considering Eq. (18), we see that the Lagrange condition is certainly satisfied at large distances. Therefore, by imposing it, we do not change the asymptotic behaviour of the solution.

Starting from the coupled-channel radial Schrödinger equation (10), it is not difficult to obtain the corresponding equations for the new unknown matrices $\mathcal{F}^{\text {(in/out })}(E, r)$. To this end, the ansatz (21) is simply substituted into Eq. (10). After substitution, the equation is transformed and simplified, using the following: firstly, the fact that matrices $W^{(\text {in/out })}(E, r)$ solve Eq. (15) (i.e. Eq. (10) without the right-hand side); secondly, the Lagrange condition (22); thirdly, introducing the diagonal matrix of the channel momenta

$$
K=\left(\begin{array}{cccc}
k_{1} & 0 & \cdots & 0 \\
0 & k_{2} & \cdots & 0 \\
\vdots & \vdots & \vdots & \vdots \\
0 & 0 & \cdots & k_{N}
\end{array}\right)
$$


and finally using known Wronskian of the Riccati-Hankel functions,

$$
W^{(\text {in })}\left[\partial_{r} W^{(\text {out })}\right]-\left[\partial_{r} W^{(\text {in })}\right] W^{(\text {out })}=2 i K .
$$

The derivation can be found in Ref. [27].

As a result, we obtain the following system of first-order differential matrix-equations

$$
\begin{aligned}
\partial_{r} \mathcal{F}^{(\mathrm{in})} & =-\frac{1}{2 i} K^{-1} W^{(\mathrm{out})} V\left[W^{(\mathrm{in})} \mathcal{F}^{(\mathrm{in})}+W^{(\mathrm{out})} \mathcal{F}^{\text {(out) }}\right], \\
\partial_{r} \mathcal{F}^{\text {(out) }} & =\frac{1}{2 i} K^{-1} W^{(\mathrm{in})} V\left[W^{(\mathrm{in})} \mathcal{F}^{(\mathrm{in})}+W^{(\text {out })} \mathcal{F}^{(\text {out })}\right]
\end{aligned}
$$

which are equivalent to the initial second-order Schrödinger equation (10). Since these equations are of the first order, the boundary conditions for them can only be imposed at a single point. A natural way of doing this is to demand that matrix (21) is regular at the origin. Then

$$
\mathcal{F}^{\text {(in) }}(E, 0)=\mathcal{F}^{\text {(out) }}(E, 0)
$$

because both $h_{\ell}^{(+)}(z)$ and $h_{\ell}^{(-)}(z)$ are singular at $z=0$, but their singularities exactly cancel each other in the combination

$$
h_{\ell}^{(+)}(z)+h_{\ell}^{(-)}(z) \equiv 2 j_{\ell}(z) .
$$

The choice of the common value for the functions in Eq. (27) determines the overall normalization of the fundamental matrix (21), because Eqs. (25|26) are linear and homogeneous. In the single-channel case, the regular solution is usually normalized in such a way that it coincides with the Riccati-Bessel function when $r \rightarrow 0$ [25]. From Eq. (28), it is clear that if we demand that our approach gives the traditional single-channel solution when $N=1$, we should use the following boundary conditions for Eqs. (25]26)

$$
\mathcal{F}^{(\text {in })}(E, 0)=\mathcal{F}^{\text {(out) }}(E, 0)=\frac{1}{2} I
$$

where $I$ is the diagonal unit matrix.

Thanks to the fact that the in-coming and out-going waves in the fundamental matrix (21) are factorized, it is easy to construct the physical solutions with proper asymptotics. For example, the bound states at large distances can have only the out-going waves. This is achieved by finding such combination coefficients in Eq. (14), i.e. in

$$
u_{n}=\sum_{n^{\prime}} \Phi_{n n^{\prime}} C_{n^{\prime}}=\sum_{n^{\prime} n^{\prime \prime}}\left[W_{n n^{\prime \prime}}^{(\mathrm{in})} \mathcal{F}_{n^{\prime \prime} n^{\prime}}^{(\mathrm{in})}+W_{n n^{\prime \prime}}^{(\text {out })} \mathcal{F}_{n^{\prime \prime} n^{\prime}}^{(\text {out })}\right] C_{n^{\prime}}
$$


that all the columns involving the in-coming waves, disappear when $r \rightarrow \infty$. In other words, for the physical wave function of a bound state, we have

$$
\sum_{n^{\prime}} \mathcal{F}_{n n^{\prime}}^{(\mathrm{in})}(E, r) C_{n^{\prime}} \underset{r \rightarrow \infty}{\longrightarrow} \sum_{n^{\prime}} F_{n n^{\prime}}^{(\mathrm{in})}(E) C_{n^{\prime}}=0
$$

This is a system of linear homogeneous equations for the unknown combination coefficients $C_{n}$. It has a non-trivial solution if and only if the corresponding determinant is zero. This results in Eq. (20) which can have solutions at discrete values of the energy. Actually, the resonant states are found in the same way, i.e. by solving the same Eq. (20), but at complex values of $\mathcal{E}_{n}$. This approach therefore offers a unified way of finding both the bound and resonant states as well as the $S$-matrix (19) for the scattering states. The corresponding physical wave functions are obtained with correct asymptotic behaviour (analytically factorized Riccati-Hankel functions).

\section{Riemann surface}

For a fixed (generally speaking, complex) value of the energy $E$, each of the $N$ channel momenta (12) can have two different values

$$
k_{n}= \pm \sqrt{\frac{2 \mu_{n}}{\hbar^{2}}\left(E-E_{n}\right)}, \quad n=1,2, \ldots, N,
$$

depending on the choice of the sign in front of the square root. All these momenta are involved as papameters in Eqs. $(25,26)$. This means that the Jost matrices $F^{\text {(in/out) }}(E)$ are not single-valued functions of $E$. At each point $E$, they have $2^{N}$ different values, for all possible combinations of the signs of $N$ channel momenta.

In complex analysis, the multi-valued functions are treated as single-valued, but defined on a multi-layered complex surface which is called Riemann surface. In our case, each layer (sheet) of this surface corresponds to a different combination of the signs of $N$ channel momenta, and thus the Riemann surface of the energy consists of $2^{N}$ sheets.

When we move around a threshold point, we go from one layer to another. Indeed, a point on a circle centered at the threshold $E_{n}$, can be parametrized as $E=E_{n}+\rho \exp (i \varphi)$, where $\rho$ is the distance from $E_{n}$ and $\varphi$ is the polar angle. The corresponding channel momentum

$$
k_{n}=\sqrt{\frac{2 \mu_{n} \rho}{\hbar^{2}}} e^{i \varphi / 2}
$$


changes its sign after one full circle and comes back to its initial value after two full circles $(\varphi=4 \pi)$. This means that the sheets of the Riemann surface are connected to each other and thus form a united multi-layer manifold. The threshold points $E_{n}(n=1,2, \ldots, N)$ are the branching points on this manifold. By moving around these points, we can continuously reach any of the $2^{N}$ sheets.

In principle, we can construct the Riemann surface rather arbitrarily by making cuts and appropriate connections of the layers. In quantum theory, it is standard that each layer is cut along the real energy axis. The cut starts at the branching point and goes to infinity in the positive direction. The edges of the cuts of different layers are interconnected in such a way that the corresponding channel momenta appropriately change their signs.

The simplest two-layer Riemann surface for the single-channel case is easy to visualize (see, Fig. 11). The two-channel problem with two branching points and four interconnected layers is much more involved. These connections for the three intervals, $E<E_{1}$, $E_{1}<E<E_{2}$, and $E>E_{2}$ are schematically shown in Fig. 2] [28]. When $N>2$, the surface is so complicated that it is not worthwhile even trying to visualize it.

In the present paper, we construct the Jost matrices in such a way that in their matrix elements the dependences on odd powers of all channel momenta are factorized analytically (see Sec. 7). The remaining matrices depend only on even powers of all the momenta $k_{n}$ and thus are single-valued functions of variable $E$. This saves us the trouble of dealing with the complicated multi-layered manifold. Moreover, using the analytically factorized dependence on $k_{n}$, we can establish some of the symmetry properties of Jost matrices, i.e. we can relate their values at some points belonging to different layers of the Riemann surface (see Sec. 8).

\section{Complex rotation}

When the potential is cut off at certain radius $R$, the right-hand sides of Eqs. (25,26) vanish for $r>R$ and the derivatives $\partial_{r} \mathcal{F}^{\text {(in/out) }}$ become zero, i.e. these functions do not change beyond this point. Therefore, in the spirit of the variable phase approach, the functions $\mathcal{F}^{\text {(in/out) }}(E, r)$ are the Jost matrices for the potential which is cut off at the point $r$. In general, when the potential asymptotically vanishes at large distances, we have

$$
\mathcal{F}^{\text {(in/out) }}(E, r) \underset{r \rightarrow \infty}{\longrightarrow} F^{(\text {in/out })}(E) \text {. }
$$


Therefore, the Jost matrices can be calculated by numerical integration of the differential equations (25) 26) from $r=0$ up to a sufficiently large radius $R$ where the limit (34) is reached within a required accuracy.

This works perfectly for real values of the energy $E$. However, when we consider complex energies (for example, in search for resonances), a technical difficulty arises. This difficulty is caused by the asymptotic behaviour of the Riccati-Hankel functions [29],

$$
h_{\ell}^{( \pm)}(k r) \underset{|k r| \rightarrow \infty}{\longrightarrow} \mp i \exp \left( \pm i k r \mp i \frac{\ell \pi}{2}\right) \text {. }
$$

As is seen, when $k$ is complex, either $h_{\ell}^{(+)}(k r)$ or $h_{\ell}^{(-)}(k r)$ exponentially diverges, depending on the sign of $\operatorname{Im} k$. As a result, either the first or the second of the equations (25]26) does not give a numerically convergent solution. This difficulty is circumvented by using the deformed integration path shown in Fig. 3, Instead of integrating the differential equations along the real axis from $r=0$ to $r=R$, we can reach the final point via the intermediate point $r=R^{\prime}$ in the complex plane. Moreover, we can safely ignore the arc $R^{\prime} R$ since the potential is practically zero at that distance.

Why does this complex rotation help? The answer can be found by looking at Eq. (35). Indeed, the asymptotic behaviour (divergent or convergent) of the functions $h_{\ell}^{( \pm)}(k r)$ is determined by the sign of $\operatorname{Im}(k r)$. If $k=|k| e^{i \varphi}$, we can always find such a rotation angle $\theta$ in $r=|r| e^{i \theta}$ that the product

$$
k r=|k r| e^{i(\varphi+\theta)}
$$

has either positive or negative (or even zero) imaginary part. Various technical details of using complex rotation in calculating the Jost functions and Jost matrices can be found in Refs. [30, 31, 32, 33, 34, 35, 36, 37, 38, 39.

\section{Factorization}

Eqs. (25,26) are very convenient for numerical calculation of the Jost matrices. However, for the purpose of power-series expansion of these matrices, we need to further transform them. The idea of such a transformation is based on the relation between the two pairs of linearly independent solutions of the Riccati-Bessel equation, namely, between the Riccati-Hankel functions $h_{\ell}^{( \pm)}$and the pair of Riccati-Bessel $j_{\ell}$ and Riccati-Neumann $y_{\ell}$ functions,

$$
h_{\ell}^{( \pm)}(z)=j_{\ell}(z) \pm i y_{\ell}(z)
$$


Introducing the diagonal matrices

$$
\begin{aligned}
J=\frac{1}{2}\left[W^{(\mathrm{in})}+W^{(\mathrm{out})}\right] & =\left(\begin{array}{cccc}
j_{\ell_{1}}\left(k_{1} r\right) & 0 & \cdots & 0 \\
0 & j_{\ell_{2}}\left(k_{2} r\right) & \cdots & 0 \\
\vdots & \vdots & \vdots & \vdots \\
0 & 0 & \vdots & j_{\ell_{N}}\left(k_{N} r\right)
\end{array}\right), \\
Y=\frac{i}{2}\left[W^{(\mathrm{in})}-W^{(\mathrm{out})}\right] & =\left(\begin{array}{cccc}
y_{\ell_{1}}\left(k_{1} r\right) & 0 & \cdots & 0 \\
0 & y_{\ell_{2}}\left(k_{2} r\right) & \cdots & 0 \\
\vdots & \vdots & \vdots & \vdots \\
0 & 0 & \vdots & y_{\ell_{N}}\left(k_{N} r\right)
\end{array}\right),
\end{aligned}
$$

as well as the new unknown matrices

$$
\begin{aligned}
& \mathcal{A}(E, r)=\mathcal{F}^{\text {(in) }}(E, r)+\mathcal{F}^{\text {(out) }}(E, r), \\
& \mathcal{B}(E, r)=i\left[\mathcal{F}^{(\text {in })}(E, r)-\mathcal{F}^{\text {(out })}(E, r)\right],
\end{aligned}
$$

we obtain another (equivalent) representation of the fundamental matrix of regular solutions,

$$
\Phi(E, r)=J(E, r) \mathcal{A}(E, r)-Y(E, r) \mathcal{B}(E, r) .
$$

Combining Eqs. (25]26), it is easy to obtain an equivalent system of differential equations for the new unknown matrices,

$$
\begin{aligned}
\partial_{r} \mathcal{A} & =-K^{-1} Y V(J \mathcal{A}-Y \mathcal{B}), \\
\partial_{r} \mathcal{B} & =-K^{-1} J V(J \mathcal{A}-Y \mathcal{B}),
\end{aligned}
$$

with the boundary conditions

$$
\mathcal{A}(E, 0)=I, \quad \mathcal{B}(E, 0)=0,
$$

which immediately follow from (29). Similarly to the limit (34), these matrices also should converge to their asymptotic values

$$
\mathcal{A}(E, r) \underset{r \rightarrow \infty}{\longrightarrow} A(E), \quad \mathcal{B}(E, r) \underset{r \rightarrow \infty}{\longrightarrow} B(E)
$$

from which the Jost matrices can be obtained,

$$
F^{(\mathrm{in})}(E)=\frac{1}{2}[A(E)-i B(E)], \quad F^{(\text {out })}(E)=\frac{1}{2}[A(E)+i B(E)] .
$$


Now, we use the fact that the Riccati-Bessel and Riccati-Neumann functions can be represented by absolutely convergent series,

$$
\begin{aligned}
& j_{\ell}(k r)=\left(\frac{k r}{2}\right)^{\ell+1} \sum_{n=0}^{\infty} \frac{(-1)^{n} \sqrt{\pi}}{\Gamma(\ell+3 / 2+n) n !}\left(\frac{k r}{2}\right)^{2 n}=k^{\ell+1} \tilde{j}_{\ell}(E, r), \\
& y_{\ell}(k r)=\left(\frac{2}{k r}\right)^{\ell} \sum_{n=0}^{\infty} \frac{(-1)^{n+\ell+1}}{\Gamma(-\ell+1 / 2+n) n !}\left(\frac{k r}{2}\right)^{2 n}=k^{-\ell} \tilde{y}_{\ell}(E, r),
\end{aligned}
$$

where we factorize the the functions $\tilde{j}_{\ell}$ and $\tilde{y}_{\ell}$, which do not depend on odd powers of $k$ and thus are single-valued functions of the energy $E$.

Let us look for the matrices $\mathcal{A}$ and $\mathcal{B}$ in the form

$$
\mathcal{A}_{i j}=\frac{k_{j}^{\ell_{j}+1}}{k_{i}^{\ell_{i}+1}} \tilde{\mathcal{A}}_{i j}, \quad \mathcal{B}_{i j}=k_{i}^{\ell_{i}} k_{j}^{\ell_{j}+1} \tilde{\mathcal{B}}_{i j},
$$

where certain powers of the channel momenta are factorized in each individual matrix element. When the representations (47, 48, 49) are substituted into Eqs. (42443), all the channel-momenta factors cancel out, and we remain with the equations for the tilded functions,

$$
\begin{aligned}
\partial_{r} \tilde{\mathcal{A}} & =-\tilde{Y} V(\tilde{J} \tilde{\mathcal{A}}-\tilde{Y} \tilde{\mathcal{B}}), \\
\partial_{r} \tilde{\mathcal{B}} & =-\tilde{J} V(\tilde{J} \tilde{\mathcal{A}}-\tilde{Y} \tilde{\mathcal{B}}),
\end{aligned}
$$

where the matrices $\tilde{J}$ and $\tilde{Y}$ differ from (37) and (38) by the diagonal factors,

$$
J=\left(\begin{array}{cccc}
k_{1}^{\ell_{1}+1} & 0 & \ldots & 0 \\
0 & k_{2}^{\ell_{2}+1} & \ldots & 0 \\
\vdots & \vdots & \vdots & \vdots \\
0 & 0 & \vdots & k_{N}^{\ell_{N}+1}
\end{array}\right) \tilde{J}, \quad Y=\left(\begin{array}{cccc}
k_{1}^{-\ell_{1}} & 0 & \ldots & 0 \\
0 & k_{2}^{-\ell_{2}} & \ldots & 0 \\
\vdots & \vdots & \vdots & \vdots \\
0 & 0 & \vdots & k_{N}^{-\ell_{N}}
\end{array}\right) \tilde{Y}
$$

The main advantage of Eqs. (50,51) is that they do not involve any coefficients or functions depending on odd powers of the channel momenta. This means that their solutions, i.e. the matrices $\tilde{\mathcal{A}}(E, r)$ and $\tilde{\mathcal{B}}(E, r)$, are single-valued functions of the energy. The multivaluedness of the initial matrices $\mathcal{A}(E, r)$ and $\mathcal{B}(E, r)$ as well as the fact that they are defined on a complicated Riemann surface, are determined by the momentum-factors separated in Eqs. (49). 


\section{Symmetry of the Jost matrices}

As an example of usefulness of the analytic structure of the Jost matrices, established in the previous section, let us consider the relation between $F^{(\text {in })}$ and $F^{(\text {out })}$. If $\tilde{A}(E)$ and $\tilde{B}(E)$ are the asymptotic values of $\tilde{\mathcal{A}}(E, r)$ and $\tilde{\mathcal{B}}(E, r)$, respectively, then according to Eqs. (46, 49), we have the following semi-analytic expressions for the Jost matrices

$$
\begin{aligned}
F_{m n}^{(\mathrm{in})} & =\frac{k_{n}^{\ell_{n}+1}}{2 k_{m}^{\ell_{m}+1}} \tilde{A}_{m n}-\frac{i k_{m}^{\ell_{m}} k_{n}^{\ell_{n}+1}}{2} \tilde{B}_{m n}, \\
F_{m n}^{(\text {out })} & =\frac{k_{n}^{\ell_{n}+1}}{2 k_{m}^{\ell_{m}+1}} \tilde{A}_{m n}+\frac{i k_{m}^{\ell_{m}} k_{n}^{\ell_{n}+1}}{2} \tilde{B}_{m n} .
\end{aligned}
$$

If we change the signs of all the channel momenta to the opposite, the matrices $\tilde{A}(E)$ and $\tilde{B}(E)$ remain unchanged while the factorized momenta generate a common factor $(-1)^{\ell_{m}+\ell_{n}}=(-1)^{\ell_{m}-\ell_{n}}$ and the sign between the two terms in Eqs. (53, 54) is also changed to the opposite. In other words,

$$
F_{m n}^{(\text {in })}\left(-k_{1},-k_{2}, \ldots,-k_{N}\right)=(-1)^{\ell_{m}+\ell_{n}} F_{m n}^{(\text {out })}\left(k_{1}, k_{2}, \ldots, k_{N}\right)
$$

where for the sake of clarity, we replaced the single independent variable $E$ with the set of channel momenta. This means that the two Jost matrices $F^{\text {(in) }}$ and $F^{\text {(out) }}$ are not completely independent. Their values at different points on the Riemann surface are the same. The $S$ matrix can be re-written as

$$
S_{m n}=(-1)^{\ell_{m}+\ell_{n}} F_{m n}^{(\mathrm{in})}\left(-k_{1},-k_{2}, \ldots,-k_{N}\right)\left[F_{m n}^{(\mathrm{in})}\left(k_{1}, k_{2}, \ldots, k_{N}\right)\right]^{-1},
$$

which is well known in the case of a single-channel problem. The symmetry property (55) is the simplest one (but perhaps the most important) and can be established in a different way (see, for example, Ref. [25]). Its simple proof is given here to merely demonstrate how the factorized semi-analytic expressions (53, 54) can be used. Establishing the other symmetry properties is beyond the scope of the present paper.

\section{Power-series expansion}

The coefficients of differential equations (50,51), i.e. the products of $\tilde{J}(E, r), \tilde{Y}(E, r)$, and the potential matrix $V$, depend on the parameter $E$, but do not involve any functions depending on channel momenta. The boundary conditions for them,

$$
\tilde{\mathcal{A}}(E, 0)=I, \quad \tilde{\mathcal{B}}(E, 0)=0,
$$


are also momentum independent. This means that the matrices $\tilde{\mathcal{A}}(E, r)$ and $\tilde{\mathcal{B}}(E, r)$ are single-valued functions of the parameter $E$, i.e. they are defined on a single sheet of the complex E-plane.

Since the matrices $\tilde{J}(E, r)$ and $\tilde{Y}(E, r)$ are holomorphic, the coefficients of differential equations (50,51) are also holomorphic for any finite radius $r$. Moreover, the boundary conditions (57) do not depend on $E$. According to the Poincaré theorem [40], this means that $\tilde{\mathcal{A}}(E, r)$ and $\tilde{\mathcal{B}}(E, r)$ are holomorphic functions of the parameter $E$ for any finite value of $r$. Therefore they can be expanded in the power series around an arbitrary point $E_{0}$ on the complex plane of the energy,

$$
\begin{aligned}
& \tilde{\mathcal{A}}(E, r)=\sum_{n=0}^{\infty}\left(E-E_{0}\right)^{n} \alpha_{n}\left(E_{0}, r\right), \\
& \tilde{\mathcal{B}}(E, r)=\sum_{n=0}^{\infty}\left(E-E_{0}\right)^{n} \beta_{n}\left(E_{0}, r\right),
\end{aligned}
$$

where the unknown expansion coefficients $\alpha_{n}$ and $\beta_{n}$ are $(N \times N)$-matrices depending not only on variable $r$ but also on the choice of the point $E_{0}$.

Eqs. (50151) involve matrices $\tilde{J}$ and $\tilde{Y}$ for which we can obtain the expansions of the same kind but with known coefficients, $\gamma_{n}$ and $\eta_{n}$,

$$
\begin{aligned}
\tilde{J}(E, r) & =\sum_{n=0}^{\infty}\left(E-E_{0}\right)^{n} \gamma_{n}\left(E_{0}, r\right), \\
\tilde{Y}(E, r) & =\sum_{n=0}^{\infty}\left(E-E_{0}\right)^{n} \eta_{n}\left(E_{0}, r\right) .
\end{aligned}
$$

Simple recurrency relations for calculating the expansion coefficients (matrices) $\gamma_{n}\left(E_{0}, r\right)$ and $\eta_{n}\left(E_{0}, r\right)$ are derived in the Appendix A.

Substituting the expansions (58, 59, 60, 61) into Eqs. (50,51), and equating the coefficients of the same powers of $\left(E-E_{0}\right)$, we obtain the following system of differential 
equations for the unknown matrices $\alpha_{n}$ and $\beta_{n}$

$$
\begin{aligned}
\partial_{r} \alpha_{n} & =-\sum_{i+j+k=n} \eta_{i} V\left(\gamma_{j} \alpha_{k}-\eta_{j} \beta_{k}\right), \\
\partial_{r} \beta_{n} & =-\sum_{i+j+k=n} \gamma_{i} V\left(\gamma_{j} \alpha_{k}-\eta_{j} \beta_{k}\right), \quad n=0,1,2, \ldots
\end{aligned}
$$

The boundary conditions (57) are independent of $E$. As is easy to see, this implies that

$$
\alpha_{n}\left(E_{0}, 0\right)=\delta_{n 0} I, \quad \beta_{n}\left(E_{0}, 0\right)=0 .
$$

In other words, all these matrices vanish at the origin, except for the matrix $\alpha_{0}$ which becomes a diagonal unit matrix at $r=0$.

Therefore for any finite radius $r$ and an arbitrary complex $E_{0}$, we have a simple procedure for calculating the expansion coefficients. What we actually need are the corresponding expansions of the matrices $\tilde{\mathcal{A}}(E, r)$ and $\tilde{\mathcal{B}}(E, r)$ when $r \rightarrow \infty$, from which the Jost matrices (46) can be constructed. In this context, taking the limit $r \rightarrow \infty$ is not a trivial procedure. Indeed, at large distances the Riccati-Bessel and Riccati-Neumann functions behave as linear combinations of the exponential functions (35). There is nothing wrong in this behaviour if the channel momenta are real. If however the energy is complex and the momenta have non-zero imaginary parts, then the matrices $\tilde{J}(E, r)$ and $\tilde{Y}(E, r)$ involve divergent exponential functions and thus are not holomorphic when $r \rightarrow \infty$.

To some extent the situation can be saved by using exponentially decaying potentials $V_{n n^{\prime}}(r) \sim \exp \left(-\lambda_{n n^{\prime}} r\right)$, which compensate the divergence of $\tilde{J}(E, r)$ and $\tilde{Y}(E, r)$ within certain domain $\mathcal{D}$ of the complex $E$-plane along its real axis. The borders of the domain $\mathcal{D}$ are determined by the requirement that none of the coefficients of Eqs. (50,51) are divergent. The behaviour of these coefficients is determined by the products $\exp \left( \pm i k_{n} r\right) \exp \left(-\lambda_{n n^{\prime}} r\right) \exp \left( \pm i k_{n^{\prime}} r\right)$. The worst case is when we have the product of the most rapidly growing exponentials stemming from the Riccati-Bessel and RiccatiNeumann functions, and the most slowly decaying element of the potential matrix. Therefore

$$
\mathcal{D}=\left\{E: \max _{n}\left|2 \operatorname{Im} \sqrt{2 \mu_{n}\left(E-E_{n}\right) / \hbar^{2}}\right|<\min _{n n^{\prime}} \lambda_{n n^{\prime}}\right\} .
$$

The faster the potential matrix decays, the wider is the domain. An example of such a domain is shown in Fig. 4 for the two-channel model used in Sec. 10, 
Therefore when $r \rightarrow \infty$, we can use the Poincaré theorem only within $\mathcal{D}$. This means that only within this domain the matrices $\tilde{\mathcal{A}}(E, \infty)$ and $\tilde{\mathcal{B}}(E, \infty)$ are holomorphic functions of the parameter $E$. A rigorous analysis (using a different method) of the analyticity domain of the multi-channel $T$-matrix for the same class of potentials is given by Motovilov in Ref. [23].

If the potential matrix $V(r)$ (or at least its long-range tail) is an analytic function of complex variable $r$ and exponentially decays along any ray $r=|r| e^{i \theta}$ within certain sector $\theta_{\min }<\theta<\theta_{\max }$ of the complex $r$-plane, then the domain of analyticity of the matrices $\tilde{\mathcal{A}}(E, \infty)$ and $\tilde{\mathcal{B}}(E, \infty)$ can be extended by using the complex rotation described in Sec. 6 .

The physically interesting domain of the $E$-plane where the expansion proposed in the present paper, can be used in practical calculations, lies on the positive real axis (scattering and reactions) and in the close vicinity below it (pronounced resonances). Therefore we can say that to all practical purposes the expansion can be done near an arbitrary point $E_{0}$.

If we denote the asymptotic values of the matrices $\alpha_{n}$ and $\beta_{n}$ as

$$
\alpha_{n}\left(E_{0}, r\right) \underset{r \rightarrow \infty}{\longrightarrow} a_{n}\left(E_{0}\right), \quad \text { and } \quad \beta_{n}\left(E_{0}, r\right) \underset{r \rightarrow \infty}{\longrightarrow} b_{n}\left(E_{0}\right)
$$

then in vicinity of any chosen point $E_{0}$ on the Riemann surface, we can obtain semianalytic expressions for the Jost matrices in the form

$$
\begin{aligned}
F_{m n}^{(\mathrm{in})} & =\sum_{j=0}^{M}\left(E-E_{0}\right)^{j}\left[\frac{k_{n}^{\ell_{n}+1}}{2 k_{m}^{\ell_{m}+1}}\left(a_{j}\right)_{m n}-\frac{i k_{m}^{\ell_{m}} k_{n}^{\ell_{n}+1}}{2}\left(b_{j}\right)_{m n}\right], \\
F_{m n}^{\text {(out) }} & =\sum_{j=0}^{M}\left(E-E_{0}\right)^{j}\left[\frac{k_{n}^{\ell_{n}+1}}{2 k_{m}^{\ell_{m}+1}}\left(a_{j}\right)_{m n}+\frac{i k_{m}^{\ell_{m}} k_{n}^{\ell_{n}+1}}{2}\left(b_{j}\right)_{m n}\right],
\end{aligned}
$$

where $M$ is the maximal power of our expansion. The non-analytic quantities in Eqs. (67, 68), i.e. the matrices $a_{j}$ and $b_{j}$, are obtained by numerical integration of differential equations (62, 63) with the boundary conditions (64) from $r=0$ to certain large value $r=R$ (in some cases, this should be done along the deformed contour shown in Fig. 3). For a given energy $E$, the choice of the sheet of the Riemann surface, where the Jost matrices are considered, is done by appropriately choosing the signs in front of the square roots (32) for calculating the channel momenta used in Eqs. (67, 68). The central point $E_{0}$ of the expansion as well as the numerically obtained matrices $a_{j}$ and $b_{j}$ are the same 
for all the layers of the Riemann surface.

In principle, the power-series expansions (67, 68) includes infinite number of terms. In practice, however, we may take into account just a few terms $(M<\infty)$ and therefore have to solve certain number of equations in the infinite system (62, 63). It should be emphasized that not all equations of this system are coupled to each other. Indeed, due to the condition $i+j+k=n$ on their right-hand sides, the equations for $\alpha_{M}$ and $\beta_{M}$ (for any $M \geqslant 0$ ) are only linked to the corresponding equations with $n<M$. For example, the first pair of equations,

$$
\begin{aligned}
& \partial_{r} \alpha_{0}=-\eta_{0} V\left(\gamma_{0} \alpha_{0}-\eta_{0} \beta_{0}\right) \\
& \partial_{r} \beta_{0}=-\gamma_{0} V\left(\gamma_{0} \alpha_{0}-\eta_{0} \beta_{0}\right)
\end{aligned}
$$

is self-contained and is not linked to any other equation of the system. The second pair is linked only to the first one, and so on. This means that by considering a finite number of equations of this system, we do not introduce a truncation error.

In principle, it is possible to find some physical meaning (scattering length, effective radius etc.) of certain combinations of the expansion coefficients $\left(a_{j}\right)_{m n}$ and $\left(b_{j}\right)_{m n}$ in Eqs. (67,68). This would require the parametrization of the corresponding $S$-matrix (19) in terms of the channel phase-shifts. This is easily done for a single channel problem (see Ref. [16]). However for a multi-channel system (even with minimal $N=2$ ) this results in rather complicated formulae which do not add any clarity to the method. Moreover, the main advantage of the proposed method consists in the fact that it can be used not only at low but at any energies (even complex) where the notions of scattering length and effective radius are meaningless anyway. This is why we think that within our method

it is not worthwhile to derive any expressions for something like multi-channel scattering length and effective radius.

\section{Numerical example}

In order to demonstrate the efficiency and accuracy of the proposed method, we do numerical calculations for a well-studied model. For this purpose, we use the two-channel potential suggested by Noro and Taylor [41]

$$
V(r)=\left(\begin{array}{cc}
-1.0 & -7.5 \\
-7.5 & 7.5
\end{array}\right) r^{2} e^{-r}
$$


which is given in arbitrary units such that $\mu_{1}=\mu_{2}=\hbar c=1$. The threshold energies for the two channels of the Noro and Taylor model are $E_{1}=0$ and $E_{2}=0.1$, and the angular momentum is zero in both channels, $\ell_{1}=\ell_{2}=0$. This potential has an attractive well in the first channel, a repulsive barrier in the second, and rather strong coupling between the channels. As a result, it generates a rich spectrum of bound and resonant states (see Fig. 51) as well as a non-trivial energy dependence of the channel and transition cross sections (see Figs. 6, 7, and 8) [27]. This model is therefore a difficult testing ground for any new method designed for describing multi-channel processes.

As a first test, we do the power series expansions of the Jost matrices (67, 68) around the point $E_{0}=5+i 0$ on the real axis and with six terms, i.e. with $M=5$. The central point of the expansion was chosen to be not far from the first resonance, where the channel cross sections have some non-trivial energy dependence. As is seen in Figs. 6, 7, and 8, the thin curves representing the approximate cross sections, reproduce the corresponding exact cross sections rather well within a wide energy interval.

Since the approximate Jost matrices (67, 68) coincide with the exact matrices on a segment of the real axis, they must be valid also at the nearby points of the complex energy surface. Comparing the exact and approximate values of $\operatorname{det} F^{(\mathrm{in})}(E)$ at complex $E$ around the point $E_{0}$ on the third sheet (--) of the Riemann surface (see Fig. 2), we found the domains within which the relative accuracy is better than $1 \%, 5 \%$, and $10 \%$. These domains are shown in Fig. 9.

It is seen that the first resonance is within the domain of $1 \%$ accuracy and therefore must be reproduced by the approximate Jost matrix. And indeed, its determinant has zero at $E=4.768178-i 0.000686$ which is very close to the exact value $E=4.768197-i 0.000710$. With more terms in the expansion, the difference becomes smaller, and with $M=10$ all the digits are the same.

The other place where we tested the expansion, was the point $E_{0}=7.5-i 2.0$ on the third sheet (--) of the Riemann surface. This point is almost in the middle between the second and the third resonances. Fig. 10 shows how the domain of $1 \%$ accuracy increases with increasing number of terms $(M=5,7,10)$ in the expansions (67, 68). As is seen in Fig. 10, even with $M=5$ both resonances are reproduced relatively well (the filled and open circles represent the exact and approximate positions of the resonances). The zeros of the exact Jost matrix determinat are at $E=7.241200-i 0.755956$ and $E=8.171217-i 3.254166$ while the expansion with $M=5$ gives $E=7.131204-i 0.768670$ and $E=8.241795-i 2.982867$. We deliberately chose the point $E_{0}$ far away from both 
resonances. If it is close to any of them, the resonance can be found very accurately.

\section{Conclusion}

In the present paper, we show that each matrix element of multi-channel Jost matrix can be written as a sum of two terms, and each term can be factorized in such a way that it assumes the form of a product of certain combination of the channel momenta $k_{n}$ times an analytic single-valued function of the energy $E$. This means that all the branching points of the Riemann energy-surface are given in the Jost matrix explicitly via the channelmomentum factors. The remaining energy-dependent factors in all its matrix elements are defined on single energy plane which does not have any branching points anymore.

For these energy-dependent functions, we derive a system of first-order differential equations. Then, using the fact that the functions are analytic, we expand them in the power series and obtain a system of differential equations that determine the expansion coefficients. A systematic procedure developed in the present paper, allows us to accurately calculate the power-series expansion of the Jost matrices practically at any point on the Riemann surface of the energy. Actually, the expansion is done for the single-valued functions of the energy, while the choice of the sheet of the Riemann surface is done by appropriately choosing the signs of the channel momenta in the momentum-dependent factors.

The expansion suggested in the present paper, makes it possible to obtain a semianalytic expression for the Jost-matrix (and therefore for the S-matrix) near an arbitrary point on the Riemann surface and thus to locate the spectral points (bound and resonant states) as the S-matrix poles. Alternatively, the expansion can be used to parametrize experimental data, where the unknown expansion coefficients are the fitting parameters. Such a parametrization will have the correct analytic properties. After fitting the data given at real energies, one can use the semi-analytic Jost matrix to search for resonances in the nearby domain of the Riemann surface. The efficiency and accuracy of the suggested expansion is demonstrated by an example of a two-channel model.

\section{APPENDIX:}




\section{A Expansion of the Riccati functions}

As is given by Eqs. (47, 48), the Riccati-Bessel and Riccati-Neumann functions can be factorized as

$$
j_{\ell}(k r)=k^{\ell+1} \tilde{j}_{\ell}(E, r), \quad y_{\ell}(k r)=k^{-\ell} \tilde{y}_{\ell}(E, r),
$$

where the tilded functions depend on $k^{2}$, i.e. on the energy. These functions are holomorphic and thus can be expanded in Taylor series at an arbitrary point $E=E_{0}$,

$$
\tilde{j}_{\ell}(E, r)=\sum_{n=0}^{\infty}\left(E-E_{0}\right)^{n} g_{\ell n}\left(E_{0}, r\right), \quad \tilde{y}_{\ell}(E, r)=\sum_{n=0}^{\infty}\left(E-E_{0}\right)^{n} t_{\ell n}\left(E_{0}, r\right),
$$

where the coefficients are given by the derivatives

$$
\begin{aligned}
& g_{\ell n}\left(E_{0}, r\right)=\frac{1}{n !}\left[\frac{d^{n}}{d E^{n}} \tilde{j}_{\ell}(E, r)\right]_{E=E_{0}}=\frac{1}{n !}\left[\frac{d^{n}}{d E^{n}} \frac{j_{\ell}(k r)}{k^{\ell+1}}\right]_{E=E_{0}}, \\
& t_{\ell n}\left(E_{0}, r\right)=\frac{1}{n !}\left[\frac{d^{n}}{d E^{n}} \tilde{y}_{\ell}(E, r)\right]_{E=E_{0}}=\frac{1}{n !}\left[\frac{d^{n}}{d E^{n}} k^{\ell} y_{\ell}(k r)\right]_{E=E_{0}} .
\end{aligned}
$$

In order to find these derivatives, we use the following relations [29]

$$
\frac{d}{d z}\left[\frac{j_{\ell}(z)}{z^{\ell+1}}\right]=-\frac{j_{\ell+1}(z)}{z^{\ell+1}} \quad \text { and } \quad \frac{d}{d z}\left[z^{\ell} y_{\ell}(z)\right]=z^{\ell} y_{\ell-1}(z) .
$$

After a simple but lengthy algebra, we finally obtain

$$
\begin{aligned}
& g_{\ell n}\left(E_{0}, r\right)=\frac{1}{n !}\left[\left(-\frac{\mu r}{\hbar^{2}}\right)^{n} \frac{j_{\ell+n}(k r)}{k^{\ell+n+1}}\right]_{E=E_{0}}, \\
& t_{\ell n}\left(E_{0}, r\right)=\frac{1}{n !}\left(\frac{\mu r}{\hbar^{2}}\right)^{n}\left[k^{\ell-n} y_{\ell-n}(k r)\right]_{E=E_{0}} .
\end{aligned}
$$

The matrices $\gamma_{n}$ and $\eta_{n}$ of Eqs. (60, 61) are diagonal with each row having the functions (77, 178) with $\mu$ and $k$ for the corresponding channel. These functions should be the same for all sheets of the Riemann surface, i.e. for any choice of the signs of channel momenta. This is so indeed since $j_{\ell}(-z)=(-1)^{\ell+1} j_{\ell}(z)$ and $y_{\ell}(-z)=(-1)^{\ell} y_{\ell}(z)$. 


\section{References}

[1] H. A. Bethe, "Theory of the Effective Range in Nuclear Scattering", Phys. Rev., 76, $38-50$ (1949)

[2] M. L. Goldberger, K. M. Watson, " Collision Theory", John Wiley \& Sons, New York, 1964.

[3] Yea-Hwang Uang, W. C. Stwalley, "The possibility of a ${ }^{4} \mathrm{He}_{2}$ bound state, effective range theory, and very low energy He-He scattering", J. Chem. phys., 76(10), 5069$5072,(1982)$.

[4] S. J. Buckman, J. Mitroy, "Analysis of low-energy electron scattering cross sections via effective-range theory", J. Phys. B: At. Mol. Opt. Phys., 22, 1365-1384, (1989).

[5] M. Marinescu, "Computation of the scattering length and effective range in molecular physics", Phys. Rev., A50, 3177-3180, (1994).

[6] F. Arnecke, H. Friedrich, J. Madronero, "Effective-range theory for quantum reflection amplitudes", PHys. Rev., A74, 062702, (2006).

[7] Z. Idziaszek, G. Karwasz, "Applicability of Modified Effective-Range Theory to positron-atom and positron-molecule scattering", Phys. Rev., A73, 064701, (2006).

[8] Z. Idziaszek, G. Karwasz, "Shape resonances in modified effective range theory for electron-molecule collisions", ArXiv, physics.atom-ph, 0708.2991, (2007).

[9] H.-W. Hammer, Dean Lee, "Causality and the effective range expansion", ArXiv, nucl-th, 1002.4603, (2010)

[10] G. J. Kynch, Proc. Phys. Soc., A65, 83, 94 (1952)

[11] B. R. Levy, J. B. Keller, J. Math. Phys., 4, 54 (1963)

[12] R. F. Dashen, J. Math. Phys., 4, 338 (1963)

[13] V. V. Babikov, Sov. J. Nucl. Phys., 1, 793-802 (1965)

[14] V. V. Babikov, Sov. Phys. Usp., 10, 271 (1967)

[15] M. Hussein, W. Li, S. Wüster, "Canonical quantum potential scattering theory", J. Phys. A: Math. Theor., 41, 475207 (2008) 
[16] S. A. Rakityansky, N. Elander, "Generalized effective-range expansion", J. Phys. A: Math. Theor. 42, 225302 (2009)

[17] M. H. Ross, G. L. Shaw, "Scattering length and effective range theory for multichannel processes", Annals of Physics: 9, 391-415, (1960).

[18] M. H. Ross, G. L. Shaw, "Multichannel effective range theory", Annals of Physics: 13, 147-186, (1961).

[19] P. Nath, G. L. Shaw, "Multichannel effective-range theory from the N/D formalism", Phys. Rev., 138 B, (3), 702, (1965).

[20] M. W. Kermode, "Effective range theory for multi-channel scattering", Nucl. Phys., A99, 605-624, (1967).

[21] D. W. L. Sprung, M. W. Kermode, S. Klarsfeld, "On the application of effective-range theory to the ${ }^{3} \mathrm{~S}_{1}-{ }^{3} \mathrm{D}_{1}$ state of the neutron-proton system", J.Phys.G: Nucl.Phys., 8, 923-935, (1982).

[22] L. Rosenberg, "Multichannel effective-range theory with long-range interactions", Phys. Rev. A57,(3), 1862-1869, (1998).

[23] A. K. Motovilov, Theor. Math. Phys. 95 (1993), 692.

[24] L. Brand, "Differential and Difference Equations", John Wiley \& Sons, Inc., New York, 1966.

[25] J. R. Taylor, "Scattering Theory", John Wiley \& Sons, New York, 1972.

[26] J. Mathews and L. R. Walker, "Mathematical Methods of Physics", W. A. Benjamin, New York, 1964.

[27] S. A. Rakityansky, N. Elander, "Analyzing the contribution of individual resonance poles of the S-matrix to two-channel scattering", Int.J.Quant.Chem., 106, 1105-1129, (2006).

[28] W. R. Frazer, A. W. Hendry, Phys. Rev., 134B, 1307 (1964).

[29] Abramowitz M and Stegun A (ed) 1964 Handbook of Mathematical Functions (Washington DC: NBS)

[30] S. A. Rakityansky, S. A. Sofianos, K. Amos. "A method of calculating the Jost function for analytic potentials", Nuovo Cim., B111, pp. 363-378 (1996). 
[31] S. A. Sofianos and S. A. Rakityansky, "Exact method for locating potential resonances and Regge trajectories", J. Phys. A: Math. Gen. 303725 (1997).

[32] S. A. Sofianos, S. A. Rakityansky, and G. P. Vermaak, "Sub-threshold resonances in few-neutron systems", Journal of Phys., G23, pp. 1619-1629 (1997).

[33] S. A. Rakityansky, S. A. Sofianos, "Jost function for coupled partial waves", Journal of Physics, A31, pp. 5149-5175 (1998).

[34] S. A. Sofianos, S. A. Rakityansky, S. E. Massen, "Jost Function for Singular Potentials", Phys. Rev. A60, pp. 337-343 (1999).

[35] S. A. Rakityansky, S. A. Sofianos, "Jost function for coupled channels", Few-Body Systems Suppl., 10, pp. 93-96 (1999).

[36] S. E. Massen, S. A. Sofianos, S. A. Rakityansky, S. Oryu, "Resonances and off-shell characteristics of effective interactions", Nucl. Phys. A654, pp. 597-611 (1999).

[37] S. A. Rakityansky, "Unified treatment of bound, scattering, and resonant states in semiconductor nano-structures", Phys. Rev. B68(19), 195320 (2003).

[38] S. A. Rakityansky, "Modified Transfer-matrix for nano-structures with arbitrary potential profile", Phys. Rev. B70(20), 205323 (2004).

[39] S. A. Rakityansky, S. A. Sofianos and N. Elander, "Pade approximation of the Smatrix as a way of locating quantum resonances and bound states", J. Phys. A: Math. Theor., 40, 14857-14869 (2007).

[40] S. Lefschetz, "Differential Equations: Geometric Theory", Interscience Publishers, New York, 1957.

[41] T. Noro, H. S. Taylor, J. Phys. B: Atom. Mol. Phys., 13, L377 (1980). 


\begin{tabular}{|r|r|r|r|}
\hline$E_{r}$ & $\Gamma$ & $\Gamma_{1}$ & $\Gamma_{2}$ \\
\hline-2.314391 & 0 & 0 & 0 \\
\hline-1.310208 & 0 & 0 & 0 \\
\hline-0.537428 & 0 & 0 & 0 \\
\hline-0.065258 & 0 & 0 & 0 \\
\hline 4.768197 & 0.001420 & 0.000051 & 0.001369 \\
\hline 7.241200 & 1.511912 & 0.363508 & 1.148404 \\
\hline 8.171217 & 6.508332 & 1.596520 & 4.911812 \\
\hline 8.440526 & 12.562984 & 3.186169 & 9.376816 \\
\hline 8.072643 & 19.145630 & 4.977663 & 14.167967 \\
\hline 7.123813 & 26.025337 & 6.874350 & 19.150988 \\
\hline 5.641023 & 33.070140 & 8.816746 & 24.253394 \\
\hline 3.662702 & 40.194674 & 10.768894 & 29.425779 \\
\hline 1.220763 & 47.339350 & 12.709379 & 34.629971 \\
\hline-1.657821 & 54.460303 & 14.624797 & 39.835506 \\
\hline-4.949904 & 61.523937 & 16.507476 & 45.016461 \\
\hline-8.635366 & 68.503722 & 18.352084 & 50.151638 \\
\hline-12.696283 & 75.378773 & 20.155213 & 55.223560 \\
\hline-17.117760 & 82.129712 & 21.915313 & 60.214399 \\
\hline
\end{tabular}

Table 1: Spectral points $E=E_{r}-i \Gamma / 2$ generated by the potential (71) and shown in Fig. 5, 


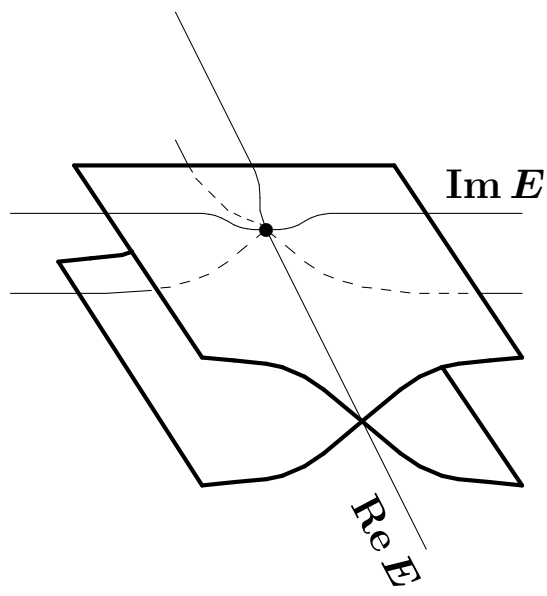

Figure 1: Riemann surface of the energy for a single-channel problem.

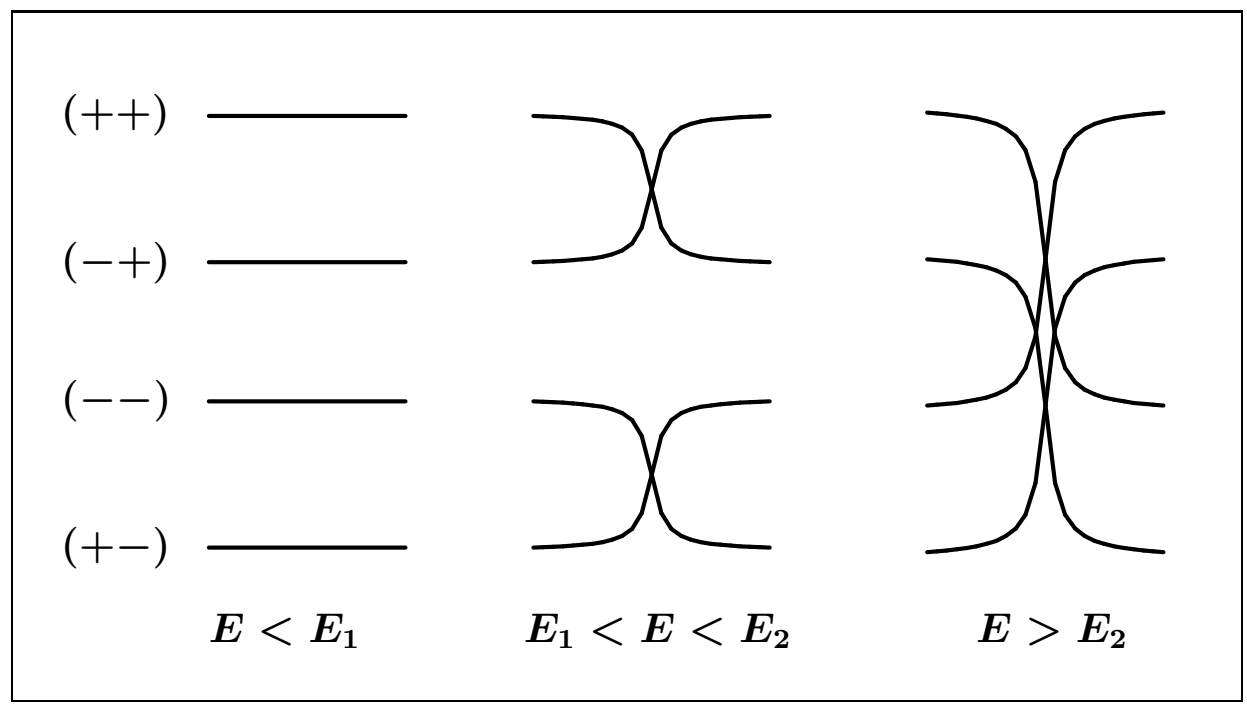

Figure 2: Schematically shown interconnections of the layers of the Riemann surface for a two-channel problem at three different energy intervals. The layers correspond to different combinations of the signs (indicated in brackets) of $\operatorname{Im} k_{1}$ and $\operatorname{Im} k_{2}$. 


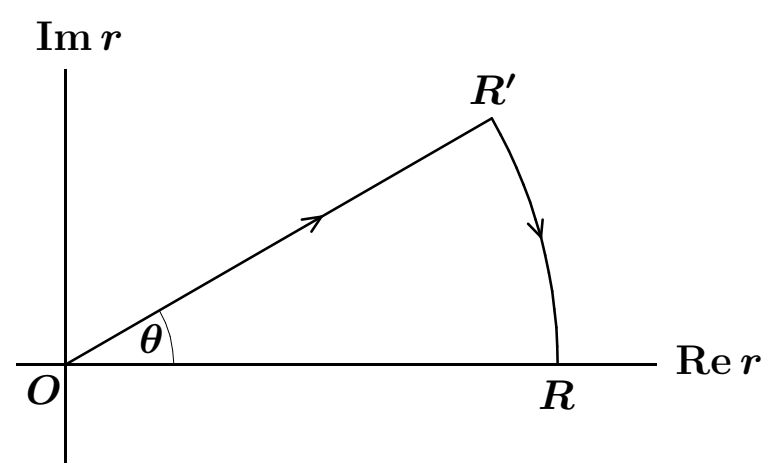

Figure 3: A deformed path for integrating the differential equations.

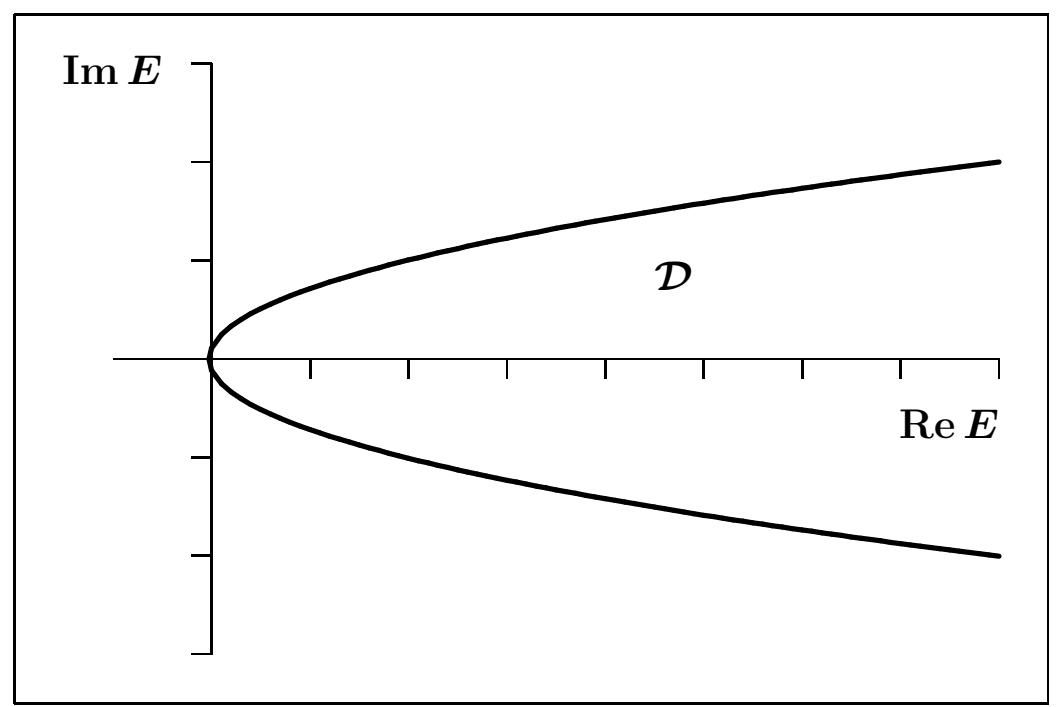

Figure 4: The domain $\mathcal{D}$ defined by Eq. (65), for the two-channel model described in Sec. 10. The matrices $\tilde{\mathcal{A}}(E, \infty)$ and $\tilde{\mathcal{B}}(E, \infty)$ for this model are holomorphic in the area enclosed between the parabolic curves. The parabola crosses the real axis at $E=-0.025$. 


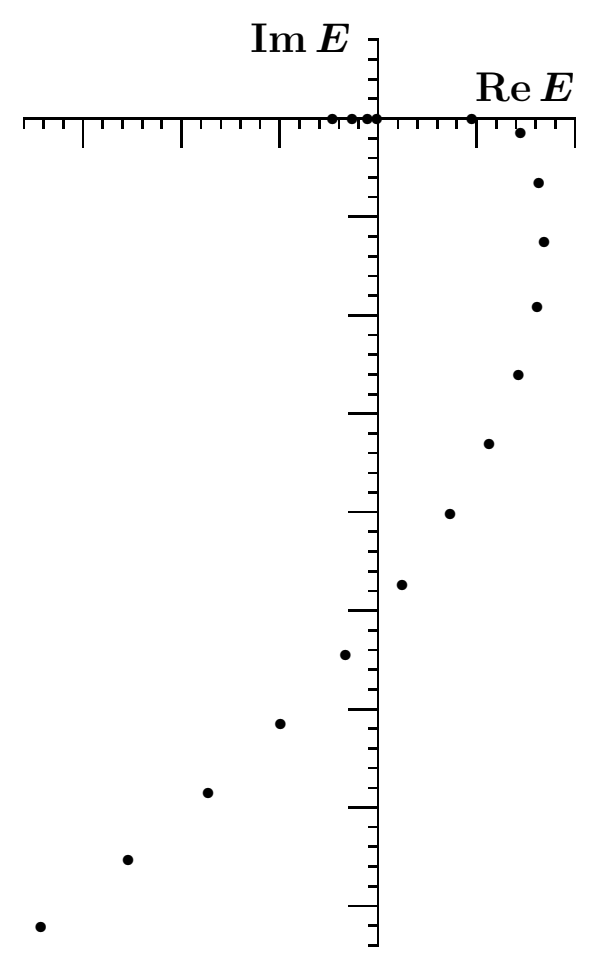

Figure 5: Spectral points generated by the potential (71) and given in Table 1 . 


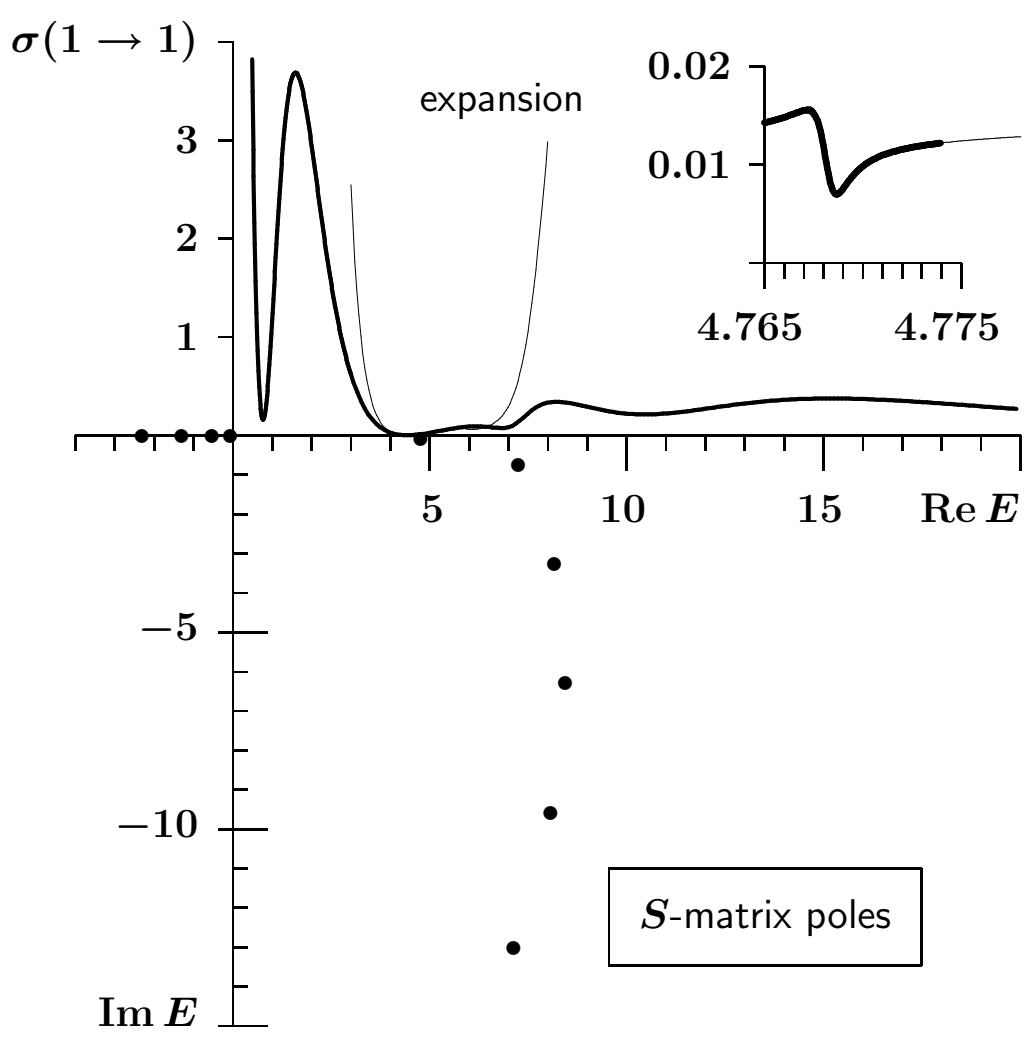

Figure 6: Energy dependence of the elastic scattering cross section in channel 1 for the potential (71). Few of the $S$-matrix poles (see Table 1 and Fig. (5)) are shown in the lower part of the Figure. The thick curve represents the exact cross section, while the thin curve shows the cross section obtained with the expansions (67, 68) where $E_{0}=5+i 0$ and $M=5$. 


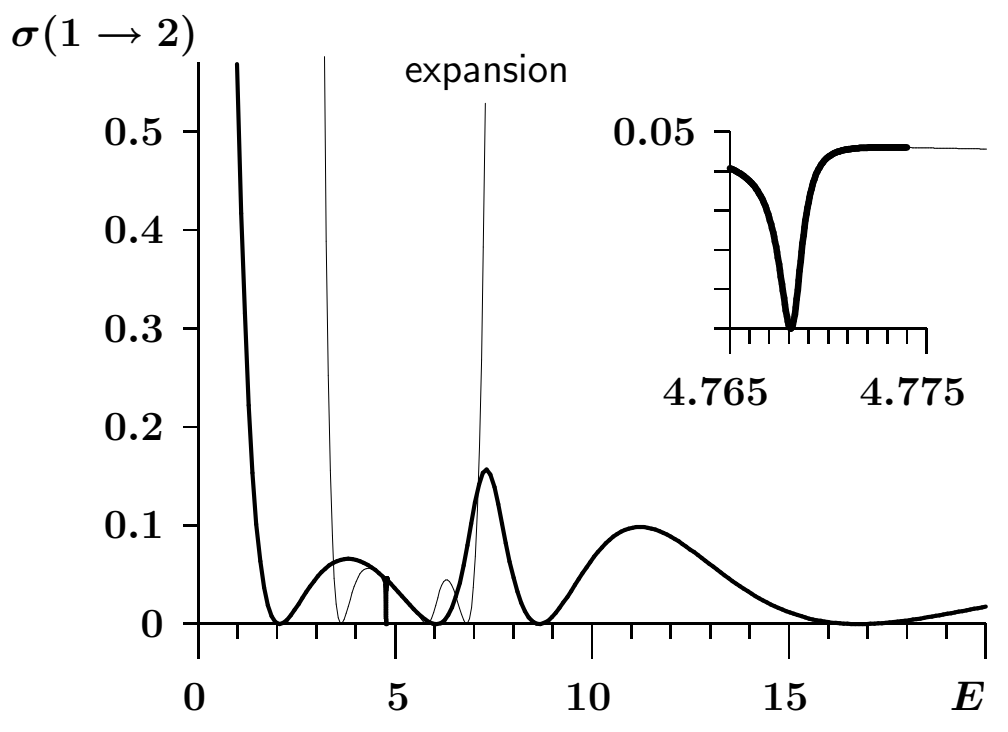

Figure 7: Cross section energy dependence of the inelastic transition $(1 \rightarrow 2)$ for the potential (71). The thick curve represents the exact cross section, while the thin curve shows the cross section obtained with the expansions (67, 68) where $E_{0}=5+i 0$ and $M=5$.

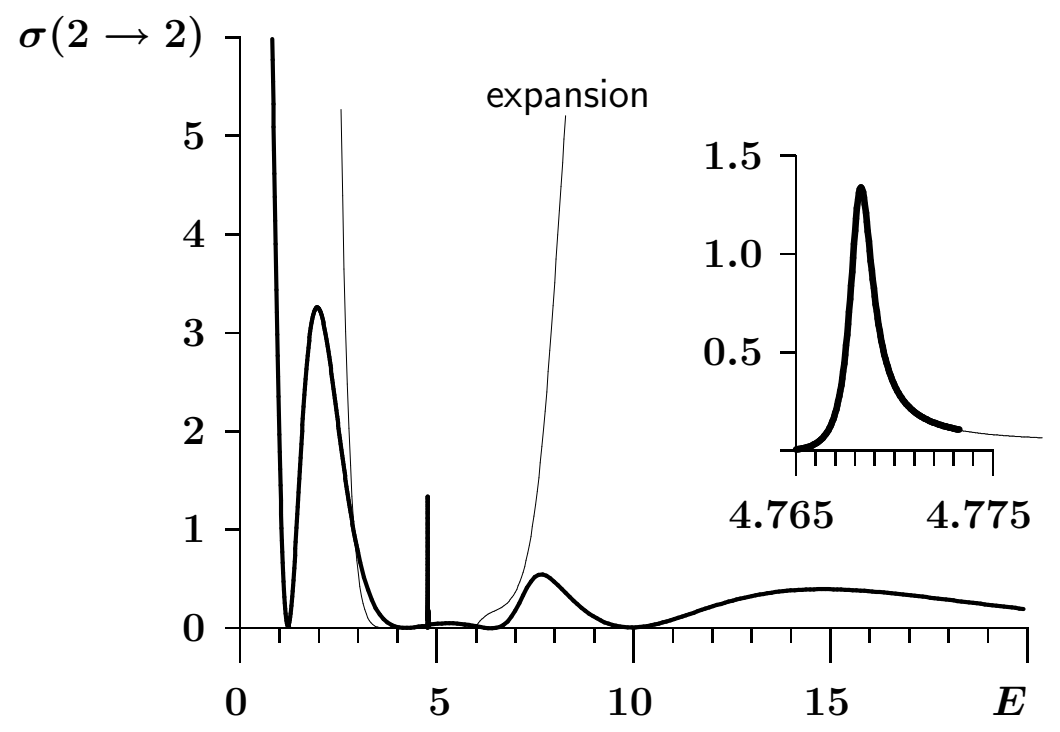

Figure 8: Energy dependence of the elastic scattering cross section in channel 2 for the potential (71). The thick curve represents the exact cross section, while the thin curve shows the cross section obtained with the expansions (67, 68) where $E_{0}=5+i 0$ and $M=5$. 


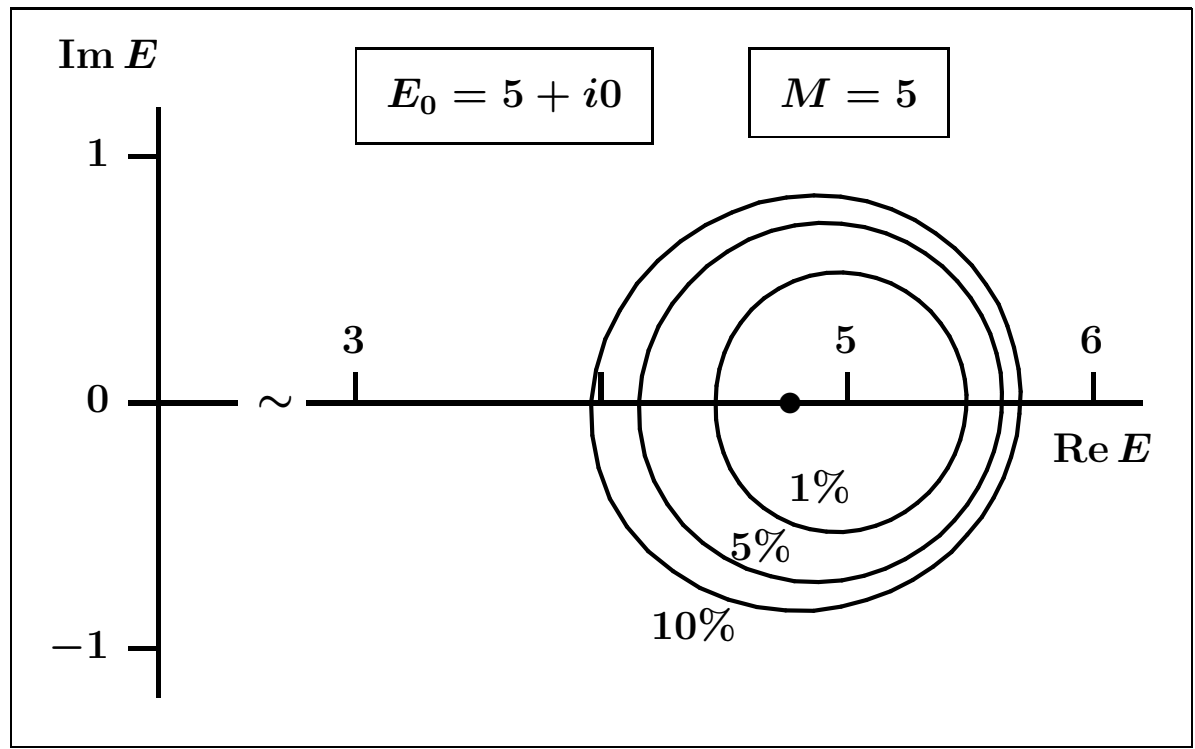

Figure 9: The domains within which the Jost matrix determinant for the potential (71) is reproduced, using the first five terms $(M=5)$ of the expansion (67), with the accuracy better than $1 \%, 5 \%$ and $10 \%$. The expansion was done around the point $E_{0}=5$ on the real axis. 


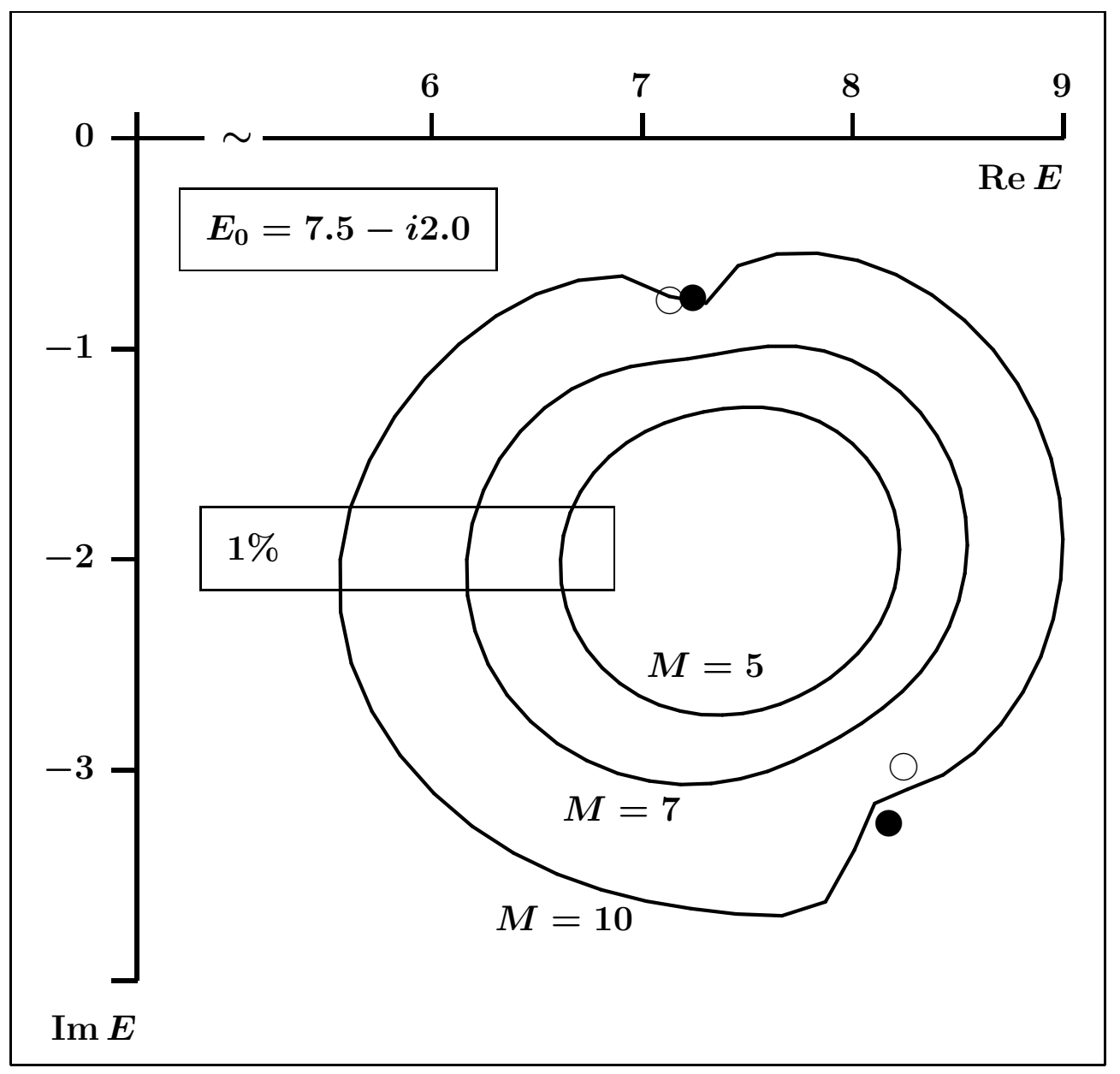

Figure 10: Growth of the 1\% accuracy domain with the increase of the number $M$ of terms in expansions (67), which were done around $E_{0}=7.5-i 2.0$ for the potential (71). Filled circles indicate the exact position of two resonances, while the open circles are their approximate positions obtained with $M=5$. 